\title{
New mechanism for the cosmological red-shift explaining: non-observation of dark energy, large-number- coincidence and the cosmic coincidence
}

\author{
Hasmukh K. Tank \\ Indian Space Research Organization, 22/693 Krishna Dahm-2, Ahmedabad 380015 India \\ E-mail: tank.hasmukh@rediffmail.com
}

\begin{abstract}
Accepting Einstein's General Relativity Theory, that the changes in the gravitational field can propagate at the speed of light, it is proposed here that: before an electron in an atom emits a photon, the energy $\left(h f_{0}\right)$ of the photon was a part of total energy of the atom; contributing to establish the gravitational-field around the atom. As soon as an electron in that atom emits a photon of energy $h f_{0}$, and the photon starts moving away from the atom, the gravitational-field around the atom partly reduces, proportional to the photon's energy $h f_{0}$, and this wave of 'reduced gravitational field' propagates radially-outwards at the speed of light. And a part of energy of the photon gets spent in "filling" the 'gravitational potential-well' produced by its energy, when it was a part of energy of the atom. From the derivation presented here we find that the energy spent by the photon to "fill" the 'gravitational potential-well', during its inter-galactic journey manifests as the "cosmological red-shift'. And the so called 'total-mass-of-the-universe" and 'radius-of-theuniverse" are just mathematically-equivalent mass and distance arising while converting electrostatic potential-energy into gravitational potential-energy. This is the reason why we find the large-number-coincidence (LNC). And since there is no expansion of the universe, there is no 'cosmic coincidence', that why only in this epoch we find the 'large-number-coincidence'!
\end{abstract}

Keywords: Cosmology; Cosmological Red-Shift; Dark-Energy; Large-Number-Coincidence; Cosmic-Coincidence.

\section{Introduction}

Alternative mechanism for the cosmological red-shift, proposed here, is not the first one; some 59 scientists have made their attempts. So this writer had proposed a criterion for short listing the likely promising explanations (Tank, Hasmukh K. 2015). At one time, this writer too tried to understand the gravitational-constant $G$ in terms of total mass $M_{0}$ and radius $R_{0}$ of the universe (Tank, Hasmukh K. 2011). Then, as the study advanced it was realized that total mass and radius of the universe are just mathematical entities, not the physically real quantities, as described in this latest finding.

Newton's gravity was 'instantaneous action at a distance." Laplace was the first to think of finite speed of gravity, as early as in 1805 . Many scientists predicted different speeds of gravity to explain the perihelion advance of Mercury. Ultimately, Einstein's proposal, that the speed of gravitational-waves too should be equal to the speed of light, got widely accepted; as it could successfully explain the perihelion advance of Mercury. Accepting Einstein's theory, that the changes in the gravitational field can propagate at the speed of light, it is proposed here that: before an electron in an atom emitted a photon, the energy $\left(h f_{0}\right)$ of the photon was a part of total energy of the atom; contributing to establish the gravitational-field around the atom. As soon an electron in that atom emits a photon of energy $h f_{0}$, and the photon starts moving away from the atom, the gravitational-field around the atom reduces proportional to the energy $h f_{0}$, and this wave of 'reduced gravitational field' propagates radially outwards at the speed of light.
And a part of energy of the photon gets spent in "filling" the 'gravitational potential-well' produced by its energy, when it was a part of energy of the atom. From the derivation presented here we find that the energy spent by the photon to "fill" the "gravitational potential-well', during its inter-galactic journey, manifests as the 'cosmological red-shift'.

\section{The derivation}

When an electron in an atom jumps from higher orbit to a lower orbit, the electrostatic potential-energy of the electron becomes more negative; and a photon of energy $h f_{0}$, equal to the difference in the potential-energy, gets emitted. Since we intend to consider here the gravitational potential-well produced by the energy of the photon, and "filling" of this potential-well from the energy of the photon, let us express this energy $h f_{0}$ in the form of gravitational potential-energy, as follows:

$\left[G M_{x}\left(h f_{0} / c^{2}\right) / R_{x}\right]=h f_{0}$

Here: $M_{x}$ and $R_{x}$ are presently unknown mass and radius for us. They will soon become known in this manuscript, as we proceed further.

Expression-1 describes the gravitational potential-energy at the point-of-emission of the photon. As the photon moves away from this point, by a distance $D$, the gravitational potential-energy of the photon reduces to the left-hand-side of expression-2, and ener- 
gy of the photon accordingly reduces to $h f$. The difference of energy gets spent in "filling" the gravitational potential-well within the spherical shell of radius $D$, up to which the gravitationalwave could reach at the speed of light.

$$
\left[G M_{x}\left(h f_{0} / c^{2}\right) /\left(R_{x}+D\right)\right]=h f
$$

Since the gravitational-force is $10^{-40}$ times weaker than the electric-force, the distance $R_{x}$ is expected to be $10^{40}$ times the reduction in radial-distance of the electron in orbit. So the conversion of electrostatic potential-energy into gravitational potential-energy is expected to obey the following relation:

(Electric-force). (Distance $\left.\mathrm{r}_{1}-\mathrm{r}_{2}\right)=($ Gravitational-force $)\left[10^{40}\left(\mathrm{r}_{1}\right.\right.$ $\left.\left.-\mathrm{r}_{2}\right)\right]$

i.e. $\left[\left(e^{2} / r_{1}^{2}\right)-\left(e^{2} / r_{2}^{2}\right)\right] .\left(r_{1}-r_{2}\right)=($ Gravitational-force $)$. $\left[10^{40}\left(r_{1}-\right.\right.$ $\left.\left.\mathrm{r}_{2}\right)\right]$

i.e. $R_{x}=\left[10^{40}\left(\mathrm{r}_{1}-\mathrm{r}_{2}\right)\right]$

Now, it will be more convenient to find the ratio, as shown in expression-3, as we intend to discover whether it is equal to the well-known ratio of the 'cosmological red-shift'. So let us find the ratio:

$\left[\left\{G M_{x}\left(h f_{0} / c^{2}\right) / R_{x}\right\}-\left\{G M_{x}\left(h f_{0} / c_{2}\right) /\left(R_{x}+D\right)\right\}\right] /\left[G M_{x}\left(h f_{0}\right.\right.$ $\left.\left./ c^{2}\right) /\left(R_{x}+D\right)\right]$

$=\left(h f_{0}-h f\right) /(h f)$

i.e. $\left(R_{x}+D-R_{x}\right)\left(R_{x}+D\right) /\left(R_{x}\right)\left(R_{x}+D\right)$

$=\left(h f_{0}-h f\right) /(h f)$

i.e. $\left(D / R_{x}\right)=\left(h f_{0}-h f\right) /(h f)$

Comparing this expression-5 with the experimentally-found expression- 6 for the 'cosmological red-shift', as per our hypothesis proposed in the introduction, that: "The energy spent by the photon to "fill"" the 'gravitational potential-well', during its intergalactic journey, manifests as the "cosmological red-shift'."

$\left(h f_{0}-h f\right) /(h f)=H_{0} D / c$

From the comparison of expressions- 5 with expression- 6 we get the value of the un-known radius $R_{x}$ as:

$\left(H_{0} D / c\right)=\left(D / R_{x}\right)$

I.e. $R_{x}=c / H_{0}=R_{0}$

Where: $R_{0}$ is currently believed to be the 'radius-of-theobservable-universe', whereas according to our hypothesis $R_{x}$ is just a distance required to express 'electrostatic potential-energy', released in the form of a photon, to express the same energy in the form of 'gravitational potential-energy'. From the known value of $R_{0}$, one can find out the value of $M_{x}$, which will be equal to the meanwhile believed 'Total-mass-of-the-universe'" $M_{0}$. And we can re-write the expression- 1 as:

$\left[G M_{0}\left(h f_{0} / c^{2}\right) / R_{0}\right]=h f_{0}$

Replacing: $\left(h f_{0} / c^{2}\right)$ with the mass of the electron $m_{e}$; and the energy $h f_{0}$ with the energy of the electron, $m_{e} c^{2}$ :

$\left[G M_{0} m_{e} / R_{0}\right]=m_{e} c^{2}$

i.e. $\left[G M_{0} m_{e} / R_{0}\right]=e^{2} / r_{e}$

Where: $e$ is electric-charge, and $r_{e}$ is classical-radius-of-theelectron. And from this expression, we arrive at P. A. M. Dirac's most familiar Large-Number-Coincidence (LNC), and its explanation by this writer (Tank, Hasmukh K. 1997), that:

$$
\begin{aligned}
& R_{0} / r_{e}=\left[G M_{0} m_{e} / e^{2}\right]=\left[G m_{p} m_{e} / e^{2}\right]\left[M_{0} / m_{p}\right] \\
& \text { i.e. }\left[R_{0} / r_{e}\right]\left[e^{2} / G m_{p} m_{e}\right]=\left[M_{0} / m_{p}\right]
\end{aligned}
$$

The Large Number Coincidence (LNC), that the ratio $\left[R_{0} / r_{e}\right]=$ $\left[e^{2} /\left(G m_{e} m_{p}\right)\right]=\left[M_{0} / m_{p}\right]^{1 / 2}$ actually implied that the "cosmological red-shift' is due to the process of conversion of electrostatic potential-energy into gravitational potential-energy, as we discussed here. Unaware of this writer's publication, Funkhouser (Nature News 2008), (Funkhouser S. 2006), (Funkhouser, S. 2008) proposed a similar explanation for LNC in 2006, with an additional question of 'cosmic coincidence', that "Why only in this epoch, when the radius of the universe is $\mathrm{R}_{0}$, we find the strength-ratio $\left(G m_{e} m_{p} / e^{2}\right)=\left(m_{\text {proton }} / M_{0}\right)^{1 / 2}=\left(r_{\text {electron }} / R_{0}\right)$ ?

Whereas as per the new mechanism for the cosmological red-shift, proposed here, we find that $M_{0}$ is not the total-mass of the universe; rather it is an "equivalent-theoretical-mass" needed to express electrostatic potential-energy in terms of 'gravitational potential-energy'; and similarly; $R_{0}$ needs not be interpreted as the 'radius-of-the-universe';'; rather $R_{0}$ is the radial-distance required while expressing 'electrostatic potential-energy' in terms of 'gravitational potential-energy'. I hope. The reader is able to appreciate this subtle difference. Finally, since there is no expansion of the universe, there is no 'cosmic coincidence', that why only in this epoch we find the 'large-number-coincidence'!

\section{Summary}

We first expressed the electrostatic potential-energy $h f_{0}$, released by an electron, while jumping from higher-orbit to a lower-orbit, in terms of gravitational potential-energy of the photon. Then, based on our hypothesis, that the part of energy of the photon gets spent in "filling" the 'gravitational potential-well' produced by the energy $h f_{0}$, we formed an expression similar to the expression for the 'cosmological red-shift'. From comparison of our expression with the experimentally-established expression for the 'cosmological red-shift' we obtained the un-known mass $M_{x}$ and un-known radius $R_{x}$; and found that they are currently believed to be the "total-mass-of-the-universe" and 'radius-of-the-universe" respectively. Then, based on our hypothesis, that: part of energy of the photon gets spent in "filling" the 'gravitational potential-well' produced by the energy $h f_{0}$, we arrived at a conclusion that "totalmass-of-the-universe" $M_{0}$ and "radius-of-the-universe" $R_{0}$ are not the 'quantities of the really physical world'; rather they are 'equivalent-theoretical-mass" and 'equivalent-radius' required to express 'electrostatic potential-energy' $h f_{0}$ in terms of 'gravitational potential-energy'. Therefore, it is not necessary that 'observable-mass-of-the-universe', (which is found to be just 5\% of $M_{0}$ ), should match with $M_{0}$; and it is not necessary that there must be 'dark energy' equal to $95 \%$ of $M_{0}$.

\section{References}

[1] Tank, Hasmukh K. (2015) "Some criteria for short listing the cosmological red-shift's ..."

www.academia.edu/.../Some_criteria_for_short_listing_the_cosmolog ical_red -shifts_explanations

[2] Tank, Hasmukh K. (2011) "Some expressions of gravity without the big 'G' - Springer link.springer.com/article/10.1007\%2Fs10509-0110891-7.

[3] Tank, Hasmukh K. (1997) "Explanation for the recurrences of LargeNumber 1040 in astrophysics" Proce. Indian Natnl, Sci. Academy- 63, a No. 6 pp 469-474 www.new1.dli.ernet.in/data1/upload/insa/INSA_2/20005975_469.pdf.

[4] Nature News http://www.nature.com/news/2008/080220/full/news.2008.610.html.

[5] Funkhouser, S. (2006) Proc. R. Soc. A 462, 2076.

[6] Funkhouser, S. (2008) doi:10.1098/rspa.2007.0370 http://dx.doi.org/10.1098/rspa.2007.0370. 